\title{
3 Research Square

\section{Mitochondrial Genome Regulator TFAM Modulates Head and Neck Tumorigenesis Through Activation of Intracellular Metabolic Reprogramming and Oncogenic Effectors}

\author{
Yi-Ta Hsieh \\ Institution of oral biology https://orcid.org/0000-0003-2160-1369 \\ Hsi-Feng Tu \\ National Yang-Ming University School of Dentistry \\ Muh-Hwa Yang \\ National Yang-Ming University \\ Yi-Fen Chen \\ National Yang-Ming University School of Dentistry \\ Chien-Ling Huang \\ The Hong Kong Polytechnic University \\ Hsin-Ming Chen \\ National Taiwan University \\ Wan-Chun Li ( $\nabla$ wcli@ym.edu.tw ) \\ National Yang-Ming University https://orcid.org/0000-0003-0481-4215
}

\section{Research}

Keywords: Head and neck cancer, Mitochondrial transcriptional factor A, Mitochondrial DNA, Metabolic reprogramming

Posted Date: September 23rd, 2020

DOl: https://doi.org/10.21203/rs.3.rs-78709/v1

License: (c) (i) This work is licensed under a Creative Commons Attribution 4.0 International License. Read Full License 


\section{Abstract}

Background: Mitochondrial defect is often observed in cancers while, in comparison with other metabolic cues, mitochondria mediated regulations in controlling tumorigenesis are less emphasized. Mitochondrial transcriptional factor A (TFAM) acts as a key regulatory factor to control mitochondrial DNA (mtDNA) replication and packing; the role of TFAM in modulating carcinogenesis, however, is controversial. Current study therefore aims to define TFAM mediated regulations in head and neck cancer (HNC) development.

Methods: Multifaceted analyses in HNC cells genetically manipulated for TFAM were performed. Clinical correlations of TFAM and its downstream Electron Transport Chain (ETC) associated factors in regulating $\mathrm{HNC}$ progression were also examined in HNC specimens and different clinical databases

Results: At the cellular level, it was demonstrated that shRNA mediated TFAM silencing resulted in an enhanced cell proliferation, both in vitro and in vivo; in contrast, TFAM overexpression suppressed cell growth. Moreover, TFAM loss also facilitated cell migration and chemodrug resistance. At the molecular basis, TFAM mediated phenotypic changes could be resulting from metabolic reprogramming by directing HNC metabolism towards aerobic glycolysis, based on the detection of less respiratory capacity in accompany with greater extracellular acidification in response to TFAM loss. Interestingly, it was also found that TFAM loss upregulated ERK1/2 and Akt-mTORC-S6 signaling activity, revealing a potential "mitochondrion-to-cytoplasm" retrograde regulatory cue in controlling HNC malignancy. Clinical impact of TFAM and its downstream targets was further examined in clinical HNC tissues while the results showed that TFAM expression and mtDNA copy numbers were significant dropped in HNC tissues compared with their normal counterparts. By using clinical databases, HNC subjects with higher TFAM expression and less genetic alteration(s) exhibited better survival rates.

Conclusion: Collectively, Current study uncovered a tumor suppressing role of TFAM and mitochondrial genome in determining HNC oncogenicity. This TFAM mediated regualtions are through intracellular metabolic reprogramming and mitochondria-to-cytoplasm cross-talk to activate oncogenic signals.

\section{Background}

The progression of malignant transformation from non-neoplastic cells to tumorous cells can be achieved only by contribution of a series of cellular events including evasion of growth suppression, sustainable proliferative capacity, enforced activation of intracellular oncogenic/angiogenic signals and deregulated energetics [1]. In the aspect of energy production, it is widely accepted that, when compared to normal cells, cancer cells are more prone to consume greater glucose thereby forming lactate by a glycolytic pathway regardless of oxygen availability [2]. Indeed, a number of studies have shown that enzymes involved in central carbon metabolism including glycolysis and Pentose Phosphate Pathway (PPP) as well as de novo lipogenesis and glutaminolysis are enhanced in tumors compared with their normal counterparts; the mitochondrial related metabolic activity, on the other hand, is often downregulated [3-6]. 
To date, most investigations focus on the role of glycolytic enzymes in regulating tumorigenesis $[7,8]$, mainly due to the fact that at molecular basis differential expression of glycolytic enzymes and glucose transporters has been shown to correlate with mutations of a classic tumor suppressor protein TP53 in most cancers [9]. The roles of mitochondrial cues in controlling cellular malignancy, however, have been under appreciated until recently. For example, mitochondrial aldehyde dehydrogenase (ALDH) could facilitate cytosolic NADH content which serves as an electron donor to trigger ATP production, thereby promoting tumor progression [10]. A high level of glutamine transporter (SLC1A5) and glutaminases (GLS), two key factors involved in the conversion of glutamine into glutamate acting as a nitrogen provider for anaplerotic flux to TriCarboxylic Acid (TCA) cycle, was detected in c-myc driven cancers [11]. Interestingly, enhanced Electron Transport Chain (ETC) activity was found in Venetoclax-resistant multiple myeloma cells while application of ETC inhibitor IACS-010759 and thenoyltrifluoroacetone (TTFA) could sensitize resistance to Venetoclax through the ATF4-BIM-NOXA pathway [12]. This suggests that mitochondrial activity may not necessarily be downregulated during tumorigenesis. In addition to metabolic enzymes or transporters, accumulating "oncometabolites" due to defective TCA cycle enzymes, such as fumarate hydratase (FH), succinate dehydrogenase (SDH) and isocitrate dehydrogenase (IDH), were also detected in tumor cells implying that deregulated mitochondrial metabolites might also underlie oncogenic transformation $[13,14]$.

Mitochondria are a double-membrane organelle that contributes to energy production in most eukaryotic organisms via various bioenergetic processes including the TCA cycle, ETC and lipid catabolism. A variety of cellular processes such as cell apoptosis, differentiation, calcium homeostasis and hormone biosynthesis occur in different mitochondrial compartments $[15,16]$. With distinct structural and crucial physiological functions, mitochondria are interestingly regarded as an independent "organism" in a cell based on the fact that mitochondria have their own genome, named mitochondrial DNA (mtDNA) [17] and that mitochondria could be horizontally transferred between cells resulting in different pathophysiological consequences [18]. In humans, mtDNA is a circular genomic material and encodes 13 ETC protein subunits[17]. At the molecular level, mitochondrial transcription factor A (TFAM) plays a key role in controlling mtDNA packing, replication and transcription [17]. In clinical studies, lower mtDNA and TFAM is associated with poorer survival in ovarian cancer [19], esophageal squamous cell carcinoma [20], colorectal cancer [21] as well as oral squamous cell carcinoma [22]. At cellular basis, decreased TFAM and mtDNA content led to lower mitochondrial activity resulting in greater lactate production, increased cell proliferation and enhanced metastatic capacity in breast cancer [23], intestinal cancer [24], esophageal cancer [20] and cisplatin (CDDP) resistant ovarian cancer [19]. A more recent study showed that TFAM mediated regulations for oncogenicity in various cancers are probably occur via the disruption of LC3-II mediated autophagy [25]. In brief, most studies suggest that impaired mitochondrial could accelerate oncogenicity in different cancers, both in vitro and in vivo.

Until now, an impact of TFAM in the development of Head and Neck Cancer (HNC) and the role of TFAM in modulating cellular, molecular and metabolic identity in HNC remains elusive. Our recent study demonstrated that manipulation of a rate-limiting factor of Pyruvate Dehydrogenase complex (PDC), and Pyruvate Dehydrogenase E1 subunit (PDHA1) that controls the metabolic fate of lactate entering into 
mitochondria, could sufficiently enhance HNC cellular malignancy [26]. This is interesting to further verify the role of mitochondrial genome and mtDNA regulator TFAM in regulating HNC malignancy.

\section{Material And Methods}

\section{Chemical, cell culture, animal and clinical tissues}

Puromycin, 3-(4,5-Dimethylthiazol-2-yl)-2,5-diphenyltetrazolium bromide (MTT), CDDP, 5-fluouracil (5-FU), Paclitaxel (Taxol) and Extracellular signal-regulated kinase 1/2 (ERK1/2) inhibitor PD98059 were purchased from Sigma-Aldrich. Protein kinase B (PKB/Akt) inhibitor MK-2206 was obtained from Selleckchem. The source and culture conditions of HNC cells from different origins as well as in HNC bearing xenografic tumor growth assay [under the approval of Institutional Animal Care and Use Committee (IACUC), National Yang-Ming University (NYMU) were described elsewhere $(26,35)$. Clinical human HNC tumors and its adjacent normal tissues were obtained from the Department of Oral Maxillofacial Surgery, NYMU Hospital under approval of the Institutional Review Boards (IRB) of NYMU Hospital (IRB\#: NYMUH 2018B003) and were preserved in RNAlater immediately after surgical resection for qRT-PCR analysis (Table S1).

\section{Establishment of TFAM deficient and overexpressing HNC cells}

Plasmids encoding small hairpin RNAs (shRNA) targeting 3'-untranslated regions (3'-UTR) of TFAM gene (shTFAM) were obtained from the National RNAi Core Facility, Academia Sinica, Taiwan (Table S2). The lentiviral vectors containing shTFAM and the control shRNA targeting Luciferase (shLuc) were produced in $293 \mathrm{~T}$ cells for infection into HNC cells by using genejuice transfection reagent. Stable knockdown cell line was cultured in medium containing $2.5 \mathrm{ug} / \mathrm{ml}$ puromycin for further experiments. For making the TFAM overexpressing vector, primers targeting wild-type TFAM gene carrying BamHI (5'CGAGGATCCACCATGGCGTTTCTCCGAAGC-3') and Notl (5'- GTAGCGGCCGCATACACTCCTCAGCACCATA$\left.3^{\prime}\right)$ restriction enzyme sequences were used to amplify full length TFAM cDNA from SAS cells, which encode wild-type TFAM, using the Platinum PCR SuperMix High Fidelity system (Invitrogen ${ }^{\text {TM }}$ ). The PCR products were then cloned into the pcDNA4/myc-HisA,B,C vector (Thermo Fisher Scientific) between BamHI and Notl. Candidate colonies were picked, amplified (Plasmid Midi kit, Geneaid) and verified before transfection. For transfection of TFAM overexpressing plasmids into HNC cells, TransFectin ${ }^{\text {TM }}$ Lipid Reagent (BIO-RAD) was utilized following the manufacturer's instructions.

\section{Cellular assays}

Cells growth was measured by both MTT assay and Trypan blue exclusion assay while the cell cycle, Annexin V-FITC based cell apoptosis, transwell-based cell migration, drug resistance assay, quantitative real-time PCR (qRT-PCR), and Western blot analysis was previously described (26). Primers for qRT-PCR analysis (Table S3) and antibodies used for Western blot are listed (Table S4). Image $\mathrm{J}$ was used to quantify protein expression. 


\section{Metabolic assays}

Lactate colorimetric/fluorometric assay kit, ATP colorimetric/fluorometric Assay kit, Pyruvate assay kit, PDH Activity Colorimetric Assay kit (Biovision) and Glucose Uptake Cell-based Assay kit (Cayman Chemical) were performed following the manufacturer's instructions. Colorimetric measurements for optical density (OD) and flow cytometrical analysis were performed using an ELISA reader (TECAN instruments, USA) and Beckman Coulter CytoFLEX, respectively, at the Instrumentation Resource Center (IRC), NYMU.

\section{Mitochondrial assays}

To determine Oxygen Consumption Rates (OCRs), Seahorse XF bioanalyzer was used following the manufacturer's instructions. The relative mtDNA copy number was measured by qRT-PCR. MitoTracker ${ }^{\text {TM }}$ Red CMXRos and Carboxy-DCFDA (both from Thermo Fisher Scientific) were used to detect mitochondrial mass and activity, respectively, using flow cytometrical analysis by Beckman Coulter CytoFLEX. Living cells were also stained with MitoTracker ${ }^{\mathrm{TM}}$ Red CMXRos, followed by fixation of $4 \%$ PFA and counterstained for nuclei by DAPI. Images were captured using Zeiss LSM880 with AiryScan at IRC, NYMU. Final images were processed using Adobe Photoshop or Photolmpact X3.

\section{Metabolomics analysis}

Cellular metabolites were analyzed using Liquid Chromatography-Mass Spectrophotometry (LC-MS) at Metabolomics Core laboratory at NYMU. In brief, 106 cells were washed with PBS and treated with LC-MS grade iced methanol and water followed by vigorous vortexing and centrifugation to remove cell debris. The supernatants were collected for LC-MS metabolic profiling analysis.

\section{Statistical analysis}

All analyses were performed using the statistical software program package Prism 5 . The differences in the clinical characteristics between two groups were analyzed by a Chi-square test and student's t-test. Differences were assumed to be significant when the $p$-value was $<0.05$.

\section{Results}

\section{Changing TFAM Expression Modulates HNC Malignancy}

To examine the roles of TFAM and its targeting mtDNA during HNC development, multiple in vitro analyses in HNC cells, with different origins in response to TFAM changes, were performed. The results showed that TFAM mRNA is reduced in tested HNC cells in contrast to normal human oral fibroblasts (OF) (Fig. 1A). Next, HNC cells deficient (Fig. 1B) or enforced expressing for TFAM (Fig. 1C) were utilized to better determine the role of TFAM in controlling HNC tumorigenicity. In addition to protein expression, decreased copy number of mtDNA encoded ETC subunits (potential TFAM targets) including ND1, ND2, ND3, ND4, ND4L, ND5, ND6 (Complex I, Fig. 1D), CytB (Complex III, Fig. 1E), Cox I, Cox II, Cox III (Complex 
IV, Fig.1F) as well as ATP8, ATP6 (Complex V, Fig. 1G) were detected by real-time PCR analysis in TFAMsilencing $\mathrm{HNC}$ cells when compared with control cells. At the translational level, while no significant changes for nuclear encoded ETC proteins (NDUFB8, SDHB, UQCRC2, ATP5A) were found in TFAM knockdown HNC cells, mtDNA encoded COXII protein was greatly down-regulated in TFAM-silencing SAS, OECM1 and HSC3 cells (Fig. 1H), highlighting the knockdown efficiency in our experimental setting. In short, shRNA mediated TFAM silencing could functionally reduce TFAM and its targeting cues in HNC cells.

Next, multifaceted cellular assays were performed in TFAM deficient and overexpressing HNC cells. By using Trypan blue exclusion and MTT assays, it was shown that TFAM knockdown led to increased HNC cell proliferation in vitro (Fig. 2A). In contrast, cell growth was downregulated in TFAM overexpressing HNC cells when compared with control cells (Fig. 2B). Similar to in vitro growth, larger HNC-bearing xenografic tumors were detected in vivo in most tested HNC cells (Fig. 2C) implying that TFAM expression is negatively associated with HNC cell growth. Further analysis found that TFAM deficiency resulted in reduced G0/G1 phase but increased distribution in both $S$ and G2M phases (Fig. 2D). Meanwhile, decreased apoptotic rate was detected in TFAM-silencing HNC cells (Fig. 2E), indicating that TFAM mediated regulation for $\mathrm{HNC}$ cell growth is due to modulation of both cell cycling and cell apoptosis.

As enhanced metastatic activity and drug resistance are also hallmarks of cancer cells [27,28], cell motility and chemodrug treatment, sensitivity was next assessed in HNC cells that are deficient for TFAM expression. By using Transwell-based migration assays, TFAM loss significantly promoted HNC cell migration when compared with control cells (Fig. 2F). As for the changes of therapeutic sensitivity in response to reduced TFAM expression in HNC cells, the half maximal inhibitory concentrations (IC50) of CDDP, 5-FU and TAXOL, the most common chemodrugs for HNC in clinic, were determined. A greater half maximal inhibitory concentration (IC50) for CDDP, 5-FU and Taxol was detected in TFAM deficient HNC cells when compared with control cells (Fig. 2G). Taken together, TFAM negatively correlated with malignancy index in HNC cells, confirming that TFAM acts as a tumor suppressing factor in HNCs.

\section{TFAM Deficiency Modulates Metabolic Plasticity in HNC cells}

Metabolic plasticity was recently demonstrated in numerous studies showing that cancers could evolve to adapt environmental stresses allowing cell survival during progression [29]. Our previous findings demonstrated that manipulations for pyruvate metabolic molecules LDHA and PDHA1 led to a metabolic shift between a wide spectrum of metabolic pathways and further analysis confirmed that this metabolic reprogramming is essential for LDAH/PDHA1 mediated malignant changes in HNC cells [26]. We herein tested whether a metabolic shift could also be responsible for TFAM mediated cellular changes. Multiple bioenergetic readouts including glucose uptake, intracellular pyruvate level, extracellular lactate production, PDH activity and intracellular ATP level in TFAM-silencing HNC cells were examined. No significant difference for glucose uptake activity and intracellular pyruvate content between TFAMsilencing and control HNC cells was detected (Fig. 3A, B) whereas increasing ATP level is detected in response to TFAM loss (Fig. 3C). Interestingly, lactate secretion was elevated (Fig. 3D) while decreased 
PDH activity, using either Western blot analysis for phosphorylation status (Ser293) of PDHA1 or colorimetric assay for PDH activity was detected (Fig. 3E, F). Mitochondrial analysis using Mitotracker red staining and Seahorse Mito-stress analyzer showed that the mitochondrial membrane potential (Fig. 3G) as well as basal and maximal respiration (Fig. $3 \mathrm{H}$ ), was down-regulated in TFAM-silencing HNC cells when compared with control cells, showing that TFAM loss could induce Warburg phenotype. Interestingly, Reactive Oxygen Species (ROS), a byproduct of Oxidative Phosphorylation (OxPhos) reaction, was increased in most TFAM-silencing HNC cells compared with control counterparts whereas mtDNA encoded COXII was negatively correlated with ROS level (Fig. 1H \& Fig. 3I). This observation revealed a possibility that TFAM loss might trigger ETC dysfunction thereby resulting in greater ROS leak and eventually increased oncogenicity.

TFAM mediated metabolic shift was further evident by a LC-MS based metabolomics analysis for glycolytic and TCA cycle metabolites. The analysis shows that only metabolites in the "payoff" phase of glycolysis including 1,3 bisphosphoglycaerate, 2-/3-phosphoglycerate, and phosphoenoalpyruvate (PEP) were decreased in TFAM-silencing HNC cells while other intracellular metabolites remain unchanged (Fig. $3 \mathrm{~J}$ and Fig.S1-2). This result could be explained as a consequence of higher glycolytic metabolism in response to TFAM loss, thereby leading to a greater consumption of glyceraldehyde-3-phosphate (G-3-P) in order to meet energetic demand. As new glucose input was not significantly altered, we further suspected that there might be alternative changes of other cellular metabolic cues, such as amino acid metabolism, that could potentially compensate deregulated mitochondrial activity in a condition of TFAM knockdown to support tumorigenic activity in HNC cells. Nevertheless, no significant changes for amino acid level were found in TFAM-silencing HNC cells when compared with control cells (Fig.S3), implying that biomolecules might be not limiting for TFAM mediated HNC oncogenic regulation. Taken together, our results demonstrated that TFAM loss facilitated HNC cell malignancy likely via intrinsic metabolic reprogramming away from mitochondrial metabolism towards to aerobic glycolysis without altering external nutrition uptake and nitrogen metabolic pathway.

\section{Oncogenic Akt and ERK Signaling Pathways Regulate TFAM Mediated HNC Oncogenicity}

In addition to metabolic changes, it is widely accepted that activation of various oncogenic pathways is essential for cancer development [30, 31]. Numerous studies have shown that Akt-mTORC and EGFRERK1/2 signaling pathways are highly expressed in HNC cells and crucial for HNC carcinogenic identity, both in vivo and in vitro [32-36]. Therefore, we investigated if Akt/ERK signals are key regulators for TFAM mediated oncogenic changes in HNC cells. Western blot analysis showed that phosphorylated Akt (Ser473), phosphorylated p44/42 MAPK ERK1/2 (Thr202/Tyr204) and mTORC pathway effector phosphorylated S6 Ribosomal Protein (Ser235/Ser236) were all upregulated in TFAM-silencing HNC cells compared with control cells (Fig. 4A). At the cellular level, IFA further confirmed that HNC cells, with reduced TFAM expression, expressed a higher amount of phosphorylated Akt/ERK/S6 proteins, indicating that TFAM mediated malignant changes could be made through the modulations of ERK1/2 and AktmTORC-S6 signaling pathways (Fig. 4B). To further define the significance of Akt/ERK signaling pathways in regulating TFAM-mediated neoplastic characteristics, ERK1/2 inhibitor PD98059 and 
PKB/Akt inhibitor MK2206 were applied in TFAM-silencing HNC cells and cell proliferation was examined. The results showed that efficient inhibition of ERK1/2 and AKT activity (Fig.S4-5) significantly abolished increased cell growth in TFAM-silencing HNC cells. Strikingly, a combinational treatment of PD98059 and MK2206 exhibited a dose-dependent synergetic effect in controlling TFAM mediated HNC cell growth (Fig. 4C). These results confirmed a novel notion that mtDNA loss in response to TFAM deficiency could trigger cytosolic signaling alteration. Interestingly, previous studies have reported that the recruitment of Akt protein to mitochondria could inactivate PDC thus resulting in downregulation of OxPhos pathway under hypoxic condition [37], further supporting a potential crosstalk between Akt signal and mitochondrial metabolism. Collectively, these findings provided an alternative scheme for development of TFAM/AktERK combinational anti-cancer therapeutic strategy for HNCs.

\section{Decreased TFAM and Its Downstream Genes in Human HNCs}

As previous studies found that TFAM and mtDNA levels were positively correlated with colorectal cancer prevalence but negatively associated with liver cancer frequency [38, 39], the association between TFAM expression and HNC oncogenicity needs further determination through additional clinical trials. To this end, the expression of TFAM and its target genes in HNC and their corresponding normal tissues was analyzed using The Cancer Genome Atlas (TCGA) based databases. The results showed a positive correlation between TFAM mRNA expression and mtDNA encoded ETC I/II/IV/V transcripts (Fig. 5A). Moreover, the TFAM mRNA level was positively correlated with mRNA expression for OxPhos factors (PDHA1, PGC1a, PPARGC1 $\beta$ ) but negatively associated with glycolytic enzymes (HK2, PFKM, PGK1) (Fig. $5 B)$. The prognostic significance of TFAM in HNC patients was also defined and it was found that patients bearing HNCs with greater TFAM expression tend to have a better Overall Survival (OS) rate (Fig. 5C). Interestingly, HNC patients with TFAM genetic alteration exhibited worse OS rate than HNC patients without TFAM alteration (Median Survival: 22.19 months vs. 56.94 months) indicating that maintenance of TFAM integrity could be essential for better prognosis in HNC patients (Fig. 5D) The expression of TFAM and its downstream targets was further examined in IRB approved paired adjacent normal $(\mathrm{N})$ and tumor $(\mathrm{T})$ clinical tissues from HNC patients ( $\mathrm{N}=18$; Table $\mathrm{S} 1)$. In agreement with database analysis, TFAM mRNA expression and copy numbers of all mtDNA encoded ETC genes were significantly downregulated in tumors compared with corresponding normal tissues, suggesting that decreased TFAM levels might be important for $\mathrm{HNC}$ development (Fig. 5E). Further analysis to define a potential association of TFAM/mtDNA encoded ETC gene expression and disease progression stratified by TNM scaling was next determined. Even though the data showed no statistical significance, all mtDNA encoded ETC genes showed a decreasing trend over a T (from T1 to T4) and N stages (from N0 to N2) (Fig.6-7), suggesting that mtDNA encoded ETC genes reversely associated with disease severity. To draw a more definite conclusion, a larger number HNC samples could be required.

\section{Discussion}

In the present study, we discovered that lower TFAM and mtDNA expression led to decreased mitochondrial activity, which elicited oncogenic pathways to promote tumor proliferation, mobility and 
therapeutic sensitivity in TFAM-silencing HNC cells. At molecular basis, we further demonstrated that ERK1/2 and Akt-mTORC-S6 pathways were highly active in TFAM-silencing HNC cells and treatment of ERK/Akt inhibitors effectively repealed TFAM-mediated malignant changes. In agreement with in vitro and in vivo findings, it was also found that TFAM and mtDNA encoded ETC genes were down-regulated in cancerous HNC tissues compared with their normal counterparts. In summary, our findings provide strong evidence showing that (1) TFAM is critical for mtDNA replication in HNCs and (2) a loss of TFAM modulated mitochondrial activity and oncogenic signals, thus resulting in up-regulating HNC malignancy.

Based on previous investigations, TFAM does not always act as a suppressor in cancers. It is also reported that when TFAM is enriched it could promote cancer cell proliferation and metastasis in bladder [40], esophageal [41], gastric [42] and colon cancers [43]. It is suspected that the discrepancy of the role of TFAM in different cancers might be attributed to distinct metabolic features in order to adapt to different tumor microenvironments [44]. Moreover, genetic mutations/polymorphisms of TFAM and its downstream mtDNA genes could also be a key factor in controlling tumor cell malignancy[45]. In agreement with former findings in non-Asian HNC subjects [46], we discovered mutations in mt-ND4 (T10873A, G11719A) in all tested HNC cell lines as well as OF. Interestingly, two additional synonymous mutations (T10915C, T11819C) in mt-ND4 were detected in SAS and FaDu cells as their roles in controlling HNC tumorigenicity remain unknown (Table S5). In addition to ND4, several nonsynonymous mutations only detected in HNC cell lines, but not OF, including A4833G in ND2, C8414T in ATP8 and T14798C in CytB were uncovered for the first time in HNC cancer cells. It would be of great interest to further define the roles of these mtDNA mutations during HNC carcinogenesis, in an attempt to find a diagnostic marker for HNCs.

Another interesting discovery from our results is that TFAM seems impacted differentially for extrinsic and intrinsic metabolic cues since no significant changes for glucose uptake, intracellular ATP and pyruvate content were detected whereas elevated aerobic glycolysis was evident in TFAM-silencing HNC cells. Results from these metabolic assays suggest that the loss of TFAM does indeed alter HNC cell metabolism, however, the reprogramming seems to be the outcome of the rewiring of intracellular metabolites but not external nutrient inputs. Furthermore, it was demonstrated that the ROS level reversely correlates with mtDNA encoded COX-II expression further verifying that mitochondrial activity is likely defective in HNC cells and ROS may play a critical role in regulating oncogenicity. Nevertheless, how TFAM-silencing HNC cells ease the accumulation of ROS to promote cell malignancy remains to be explored.

It is widely accepted that cancers are metabolically dynamic in order to survive in different environmental challenges. It therefore becomes convincing that targeting tumor metabolic plasticity could be capable of improving therapeutic efficacy of anti-cancer schemes. In agreement with this concept, we found that suppression of TFAM-silencing induced ERK1/2 and Akt-mTORC-S6 activity by commercial inhibitors could effectively abolish cell viability in highly-proliferating TFAM-silencing HNC cells, implying that targeting intracellular molecular cues, in combination with metabolic manipulation, could likely develop a more efficient treatment regimen in clinical studies. In addition to intracellular molecular changes, 
mitochondrial manipulation could also result in metabolic alterations in different cancers. By taking advantage of transcriptomic analysis, previous studies found that TFAM loss led to increased angiogenesis and invasion as well as genes relates to amino acid metabolism (e.g. SLC1A5 (ASCT2) and SLC1A4 (ASCT1)) in melanoma cells [44]. Another very recent study demonstrated that BTB and CNC homology $1(\mathrm{BACH} 1)$ depletion up-regulated ETC gene expression and mitochondrial respiration, thus promoting breast cancer growth while the addition of ETC inhibitor metformin could more effectively inhibit cell survival of these ETC hyperactive breast cancers [47]. These data suggested that, in order to gain better anti-cancer therapeutic efficacy, it is important to define molecular and metabolic features upon mitochondrial manipulation.

TFAM regulates mtDNA replication and transcription in cooperating with other molecules including mitochondrial RNA polymerase (POLRMT), mitochondrial polymerase catalytic subunit- $\gamma$ ( 140 or POLG) and transcription factor B1 (TFB1M)/B2(TFB2M) [17]. Recent studies revealed that high expression of TFAM, POLG, TFB1M and TFB2M is positively associated with better prognosis in astrocytoma and Glioblastoma Multiforme [48]. On the other hand, it was found that down-regulation of POLRMT decreased mtDNA content, OxPhos activity and cell viability in acute myeloid leukemia [49]. It was also reported that TFB2M is enriched in patients with hepatocellular carcinoma as TFB2M knockdown reduced cell viability and metastasis both in vivo and in vitro [50]. While no significant correlations between TFAM and these assemble factors in HNC patients (Fig. S9), the significance of these mtDNA assembling factors in regulating HNC malignancy remained to be verified. Moreover, how TFAM interacts with these factors to control downstream target genes leading to distinct malignant changes is of great interest for further work. Future work to define TFAM directed targets using Chromatin immunoprecipitation sequencing (ChIP-Seq) analysis is currently in progress in order to better determine key downstream targets that control TFAM mediated regulations in HNC cells.

\section{Conclusions}

In summary, we highlighted the prominent role of TFAM in regulating HNC malignancy and confirmed that TFAM could be a key tumor suppressor during HNC tumorigenesis, not only in experimental setting but also in clinical specimen. In line with this finding, further work to explore small molecules or compounds to activate expression of TFAM or mitochondrial ETC encoded subunits could potentially provide an effective therapeutic option to lessen HNC malignancy.

\section{Abbreviations}

5-FU: 5-fluouracil; ALDH: Aldehyde dehydrogenase; BACH1: BTB and CNC homology 1; CDDP: Cisplatin; ChIP-Seq: Chromatin immunoprecipitation sequencing; ERK: Extracellular signal-regulated kinase; ETC:Electron Transport Chain; FH: Fumarate hydratase; GLS: Glutaminase; HNC: Head and Neck Cancer; IC50: Half maximal inhibitory concentration; IDH: Isocitrate dehydrogenase; LC-MS: Liquid Chromatography-Mass Spectrophotometry; TFAM: Mitochondrial transcriptional factor A; mtDNA: Mitochondrial DNA; MTT: 3-(4,5-Dimethylthiazol-2-yl)-2,5-diphenyltetrazolium bromide; OF: Normal 
human oral fibroblasts; OS: Overall Survival; PDC: Pyruvate dehydrogenase complex; PDHA1: Pyruvate Dehydrogenase E1 component a subunit; PEP: Phosphoenolpyruvate; PKB/Akt: Protein kinase B; POLG: Mitochondrial polymerase catalytic subunit-y; POLRMT: Mitochondrial RNA polymerase; PPP: Pentose Phosphate Pathway; ROS: Reactive oxygen species; shRNA: short-hairpin RNA; SDH: Succinate dehydrogenase; TAXOL: Paclitaxel; TCA: TriCarboxylic Acid; TCGA: The Cancer Genomic Atlas; TFB1M/TFB2M: Transcription factor B1 /B2; TTFA: ThenoylTriFluoroAcetone

\section{Declarations}

\section{Ethics approval and consent to participate}

The present study was approved by the Institutional Review Boards (IRB) of NYMU Hospital (IRB\#: NYMUH 2018B003). Animal experiment was approved by the Institutional Animal Care and Use Committee (IACUC), National Yang-Ming University (NYMU).

\section{Consent for publication}

Not applicable.

\section{Availability of data and materials}

The raw data used and analyzed in the current study are available from the corresponding author upon a reasonable request

\section{Competing interests}

The authors disclose no conflict of interest

\section{Funding}

This work is supported by grants Most-106-2314-B-010-005-MY3 and Most-109-2628-B-010-008- from Ministry of Science and Technology, Taiwan.

\section{Authors' contributions}

W-C L conceived the study and participated in the study design and coordination; Y-T H and Y-F C performed experiments; $\mathrm{H}-\mathrm{F}$ T, M-H Y and H-M C provided clinical samples; $\mathrm{Y}-\mathrm{T} \mathrm{H}, \mathrm{C}-\mathrm{L} \mathrm{H}$ and $\mathrm{W}-\mathrm{C} \mathrm{L}$ discussed and interpreted data; Y-T H performed statistical analysis; $\mathrm{Y}-\mathrm{T} \mathrm{H}$ and $\mathrm{W}-\mathrm{C} \mathrm{L}$ wrote the manuscript. All authors read and approved the final manuscript.

\section{Acknowledgements}

Authors would like to thank Professor Kuo-Wei Chang (National Yang-Ming University) for providing experimental materials. We thank the National RNAi Core Facility at Academia Sinica, Taiwan for 
providing shRNA reagents. We also thank Dr Jude Clapper (Taipei American School) for critical review and English corrections for the manuscript.

\section{Reference}

1. Hanahan D, Weinberg RA. Hallmarks of cancer: the next generation. Cell. 2011;144(5):646-74.

2. Warburg O. On the origin of cancer cells. Science. 1956;123(3191):309-14.

3. DeBerardinis RJ, Chandel NS. Fundamentals of cancer metabolism. Sci Adv. 2016;2(5):e1600200.

4. Cannino G, Ciscato F, Masgras I, Sanchez-Martin C, Rasola A. Metabolic Plasticity of Tumor Cell Mitochondria. Front Oncol. 2018;8:333.

5. Vander Heiden MG, Cantley LC, Thompson CB. Understanding the Warburg effect: the metabolic requirements of cell proliferation. Science. 2009;324(5930):1029-33.

6. Torresano L, Nuevo-Tapioles C, Santacatterina F, Cuezva JM. Metabolic reprogramming and disease progression in cancer patients. Biochim Biophys Acta Mol Basis Dis. 2020;1866(5):165721.

7. Li WC, Huang CH, Hsieh YT, Chen TY, Cheng LH, Chen CY, Liu CJ, Chen HM, Huang CL, Lo JF, et al. Regulatory Role of Hexokinase 2 in Modulating Head and Neck Tumorigenesis. Front Oncol. 2020;10:176.

8. Hsieh YT, Chen YF, Lin SC, Chang KW, Li WC. Targeting Cellular Metabolism Modulates Head and Neck Oncogenesis. Int J Mol Sci. 2019;20(16).

9. Liu J, Zhang C, Hu W, Feng Z. Tumor suppressor p53 and its mutants in cancer metabolism. Cancer Lett. 2015;356(2 Pt A):197-203.

10. Kang JH, Lee SH, Hong D, Lee JS, Ahn HS, Ahn JH, Seong TW, Lee CH, Jang H, Hong KM, et al. Aldehyde dehydrogenase is used by cancer cells for energy metabolism. Exp Mol Med. 2016;48(11):e272.

11. Stine ZE, Walton ZE, Altman BJ, Hsieh AL, Dang CV. MYC, Metabolism, and Cancer. Cancer Discov. 2015;5(10):1024-39.

12. Bajpai R, Sharma A, Achreja A, Edgar CL, Wei C, Siddiqa AA, Gupta VA, Matulis SM, McBrayer SK, Mittal A, et al. Electron transport chain activity is a predictor and target for venetoclax sensitivity in multiple myeloma. Nat Commun. 2020;11(1):1228.

13. Yang M, Soga T, Pollard PJ. Oncometabolites: linking altered metabolism with cancer. J Clin Invest. 2013;123(9):3652-8.

14. Bardella C, Pollard PJ, Tomlinson I. SDH mutations in cancer. Biochim Biophys Acta. 2011;1807(11):1432-43.

15. Friedman JR, Nunnari J. Mitochondrial form and function. Nature. 2014;505(7483):335-43.

16. Fernie AR, Carrari F, Sweetlove LJ. Respiratory metabolism: glycolysis, the TCA cycle and mitochondrial electron transport. Curr Opin Plant Biol. 2004;7(3):254-61. 
17. Gaspari M, Larsson NG, Gustafsson CM. The transcription machinery in mammalian mitochondria. Biochim Biophys Acta. 2004;1659(2-3):148-52.

18. Torralba D, Baixauli F, Sanchez-Madrid F. Mitochondria Know No Boundaries: Mechanisms and Functions of Intercellular Mitochondrial Transfer. Front Cell Dev Biol. 2016;4:107.

19. Kleih M, Bopple K, Dong M, Gaissler A, Heine S, Olayioye MA, Aulitzky WE, Essmann F. Direct impact of cisplatin on mitochondria induces ROS production that dictates cell fate of ovarian cancer cells. Cell Death Dis. 2019;10(11):851.

20. Masuike Y, Tanaka K, Makino T, Yamasaki M, Miyazaki Y, Takahashi T, Kurokawa Y, Nakajima K, Mori M, Doki Y. Esophageal squamous cell carcinoma with low mitochondrial copy number has mesenchymal and stem-like characteristics, and contributes to poor prognosis. PLoS One. 2018;13(2):e0193159.

21. Cui H, Huang P, Wang Z, Zhang Y, Zhang Z, Xu W, Wang X, Han Y, Guo X. Association of decreased mitochondrial DNA content with the progression of colorectal cancer. BMC Cancer. 2013;13:110.

22. Takeda D, Hasegawa T, Ueha T, Sakakibara A, Kawamoto T, Minamikawa T, Sakai Y, Komori T. Decreased mitochondrial copy numbers in oral squamous cell carcinoma. Head Neck. 2016;38(8):1170-5.

23. Balliet RM, Capparelli C, Guido C, Pestell TG, Martinez-Outschoorn UE, Lin Z, Whitaker-Menezes D, Chiavarina B, Pestell RG, Howell A, et al. Mitochondrial oxidative stress in cancer-associated fibroblasts drives lactate production, promoting breast cancer tumor growth: understanding the aging and cancer connection. Cell Cycle. 2011;10(23):4065-73.

24. Woo DK, Green PD, Santos JH, D'Souza AD, Walther Z, Martin WD, Christian BE, Chandel NS, Shadel GS. Mitochondrial genome instability and ROS enhance intestinal tumorigenesis in APC(Min/+) mice. Am J Pathol. 2012;180(1):24-31.

25. Jiang X, Wang J. Knockdown of TFAM in Tumor Cells Retarded Autophagic Flux through Regulating p53 Acetylation and PISD Expression. Cancers (Basel). 2020;12(2).

26. Chen TY, Hsieh YT, Huang JM, Liu CJ, Chuang LT, Huang PC, Kuo TY, Chia HY, Chou CY, Chang CW, et al. Determination of Pyruvate Metabolic Fates Modulates Head and Neck Tumorigenesis. Neoplasia. 2019;21(7):641-52.

27. Paul CD, Mistriotis P, Konstantopoulos K. Cancer cell motility: lessons from migration in confined spaces. Nat Rev Cancer. 2017;17(2):131-40.

28. Housman G, Byler S, Heerboth S, Lapinska K, Longacre M, Snyder N, Sarkar S. Drug resistance in cancer: an overview. Cancers (Basel). 2014;6(3):1769-92.

29. Keibler MA, Wasylenko TM, Kelleher JK, lliopoulos O, Vander Heiden MG, Stephanopoulos G. Metabolic requirements for cancer cell proliferation. Cancer Metab. 2016;4:16.

30. Chen YC, Hunter DJ. Molecular epidemiology of cancer. CA Cancer J Clin. 2005;55(1):45-54; quiz 7.

31. Malhotra J. Molecular and genetic epidemiology of cancer in low- and medium-income countries. Ann Glob Health. 2014;80(5):418-25. 
32. Yun MR, Choi HM, Kang HN, Lee Y, Joo HS, Kim DH, Kim HR, Hong MH, Yoon SO, Cho BC. ERKdependent IL-6 autocrine signaling mediates adaptive resistance to pan-PI3K inhibitor BKM120 in head and neck squamous cell carcinoma. Oncogene. 2018;37(3):377-88.

33. Lin X, Liao J, Yang Z, Fan X, Cullen KJ, Chen L, Dan H. Inhibition of cisplatin-resistant head and neck squamous cell carcinoma by combination of Afatinib with PD0325901, a MEK inhibitor. Am J Cancer Res. 2019;9(6):1282-92.

34. Silva-Oliveira RJ, Melendez M, Martinho O, Zanon MF, de Souza Viana L, Carvalho AL, Reis RM. AKT can modulate the in vitro response of HNSCC cells to irreversible EGFR inhibitors. Oncotarget. 2017;8(32):53288-301.

35. Liu CJ, Chang WJ, Chen CY, Sun FJ, Cheng HW, Chen TY, Lin SC, Li WC. Dynamic cellular and molecular modulations of diabetes mediated head and neck carcinogenesis. Oncotarget. 2015;6(30):29268-84.

36. Tan FH, Bai Y, Saintigny P, Darido C. mTOR Signalling in Head and Neck Cancer: Heads Up. Cells. 2019;8(4).

37. Chae YC, Vaira V, Caino MC, Tang HY, Seo JH, Kossenkov AV, Ottobrini L, Martelli C, Lucignani G, Bertolini I, et al. Mitochondrial Akt Regulation of Hypoxic Tumor Reprogramming. Cancer Cell. 2016;30(2):257-72.

38. Hu L, Yao X, Shen Y. Altered mitochondrial DNA copy number contributes to human cancer risk: evidence from an updated meta-analysis. Sci Rep. 2016;6:35859.

39. Wen S, Gao J, Zhang L, Zhou H, Fang D, Feng S. p53 increase mitochondrial copy number via upregulation of mitochondrial transcription factor $A$ in colorectal cancer. Oncotarget. 2016;7(46):7598195.

40. Mo M, Peng F, Wang L, Peng L, Lan G, Yu S. Roles of mitochondrial transcription factor $A$ and microRNA-590-3p in the development of bladder cancer. Oncol Lett. 2013;6(2):617-23.

41. Lin CS, Lee HT, Lee SY, Shen YA, Wang LS, Chen YJ, Wei YH. High mitochondrial DNA copy number and bioenergetic function are associated with tumor invasion of esophageal squamous cell carcinoma cell lines. Int J Mol Sci. 2012;13(9):11228-46.

42. Lee WR, Na H, Lee SW, Lim WJ, Kim N, Lee JE, Kang C. Transcriptomic analysis of mitochondrial TFAM depletion changing cell morphology and proliferation. Sci Rep. 2017;7(1):17841.

43. Sun X, Zhan L, Chen Y, Wang G, He L, Wang Q, Zhou F, Yang F, Wu J, Wu Y, et al. Increased mtDNA copy number promotes cancer progression by enhancing mitochondrial oxidative phosphorylation in microsatellite-stable colorectal cancer. Signal Transduct Target Ther. 2018;3:8.

44. Araujo LF, Siena ADD, Placa JR, Brotto DB, Barros, II, Muys BR, Biagi CAO, Jr., Peronni KC, Sousa JF, Molfetta GA, et al. Mitochondrial transcription factor A (TFAM) shapes metabolic and invasion gene signatures in melanoma. Sci Rep. 2018;8(1):14190.

45. Chatterjee A, Mambo E, Sidransky D. Mitochondrial DNA mutations in human cancer. Oncogene. 2006;25(34):4663-74. 
46. Allegra E, Garozzo A, Lombardo N, De Clemente M, Carey TE. Mutations and polymorphisms in mitochondrial DNA in head and neck cancer cell lines. Acta Otorhinolaryngol Ital. 2006;26(4):185-90.

47. Bader DA, Hartig SM, Putluri V, Foley C, Hamilton MP, Smith EA, Saha PK, Panigrahi A, Walker C, Zong $L$, et al. Mitochondrial pyruvate import is a metabolic vulnerability in androgen receptor-driven prostate cancer. Nat Metab. 2019;1(1):70-85.

48. Correia RL, Oba-Shinjo SM, Uno M, Huang N, Marie SK. Mitochondrial DNA depletion and its correlation with TFAM, TFB1M, TFB2M and POLG in human diffusely infiltrating astrocytomas. Mitochondrion. 2011;11(1):48-53.

49. Bralha FN, Liyanage SU, Hurren R, Wang X, Son MH, Fung TA, Chingcuanco FB, Tung AY, Andreazza $A C$, Psarianos $P$, et al. Targeting mitochondrial RNA polymerase in acute myeloid leukemia. Oncotarget. 2015;6(35):37216-28.

50. Geng X, Geng Z, Li H, Zhang Y, Li J, Chang H. Over-expression of TFB2M facilitates cell growth and metastasis via activating ROS-Akt-NF-kappaB signalling in hepatocellular carcinoma. Liver Int. 2020.

\section{Figures}




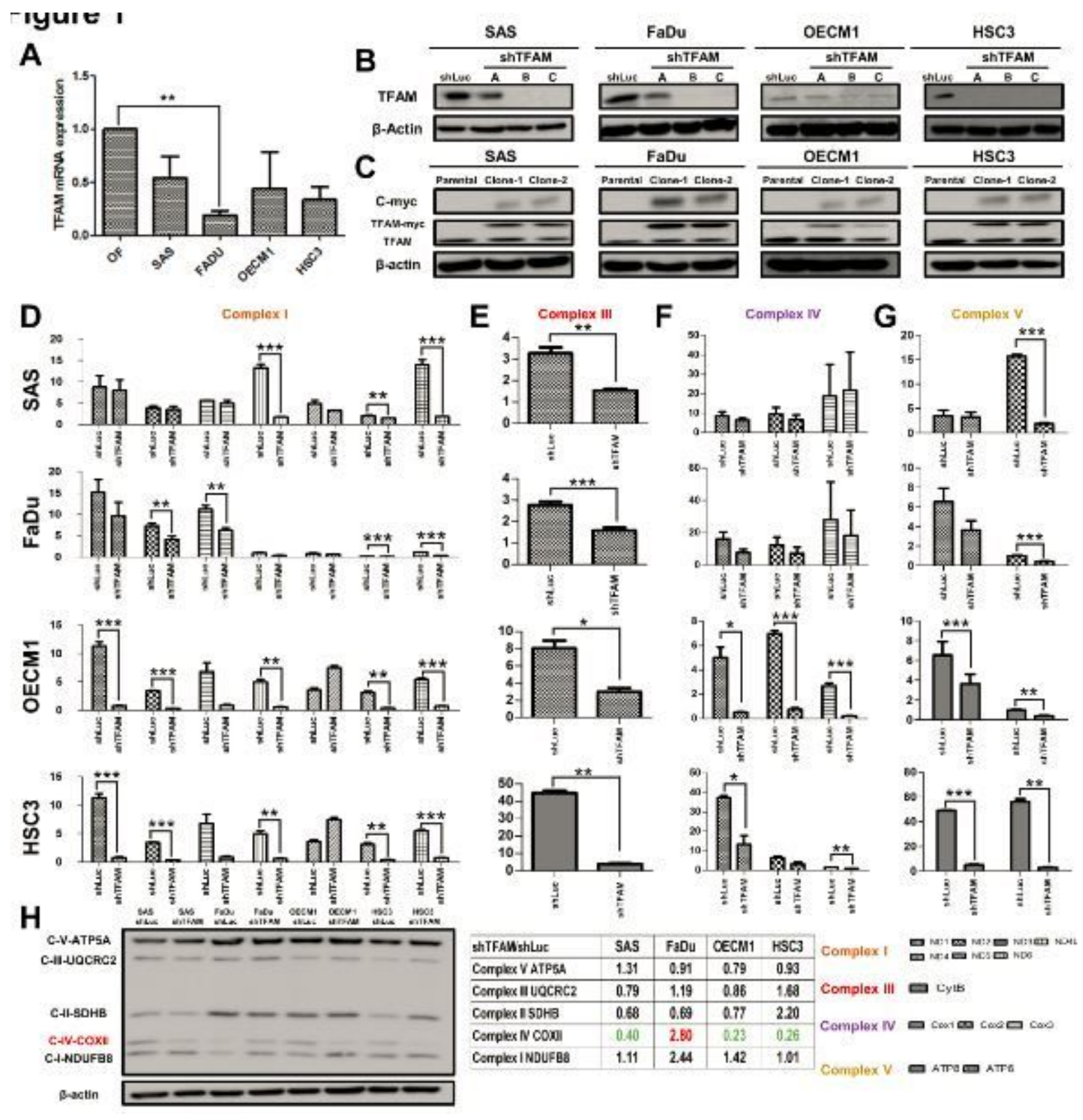

Figure 1

TFAM Alterations Lead to Functional mtDNA Change in HNC Cells (A) Real-time RT-PCR analysis showed decreased TFAM mRNA expression in HNC cells compared with OFs. Western blot analysis showed effective changes of TFAM expression in (B) shRNA-mediated TFAM silencing and (C) TFAM overexpressing HNC cells. (D-G) Functional knockdown is evident using real-time RT-PCR analysis showing significant downregulation of mtDNA-encoded ETC subunit genes in TFAM silencing HNC cells. Different ETC complex genes including Complex I (D, ND1, ND2, ND3, ND4, ND4L, ND5, ND6), Complex III (E, CytB), Complex IV (F, Cox I, Cox II, Cox III) and Complex V (G, ATP8, ATP6) are indicated. (H) Western blot analysis showed differential expression of respiratory proteins in TFAM silencing HNC cells. Quantification is represented as the fold changes of respiratory protein expression in TFAM silencing (shTFAM) over control (shLuc) HNC cells. Data are presented as Mean $\pm S E M(N>=3) .{ }^{*} p<0.05,{ }^{* \star} p<0.01$, $\star \star \star x<0.001$. 


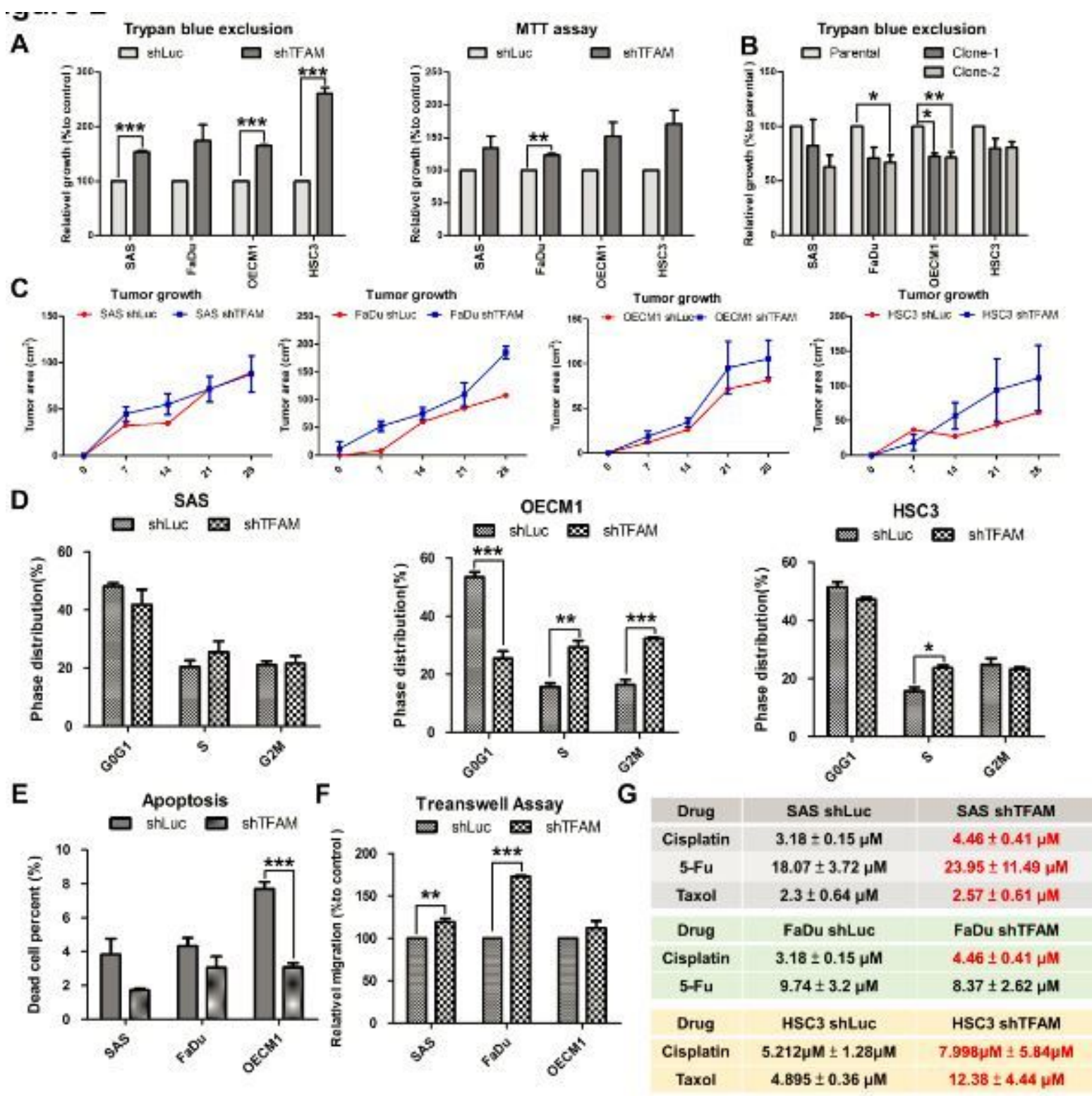

Figure 2

TFAM Loss Facilitates HNC Malignancy (A) Increased cell growth was detected in TFAM silencing HNCs cells using Trypan exclusion assay and MTT assay compared with control cells. (B) On the contrary, HNC cells enforced expressing TFAM showed a decreased cell growth compared with their parental counterparts. (C) In vivo analysis for HNC-bearing xenografic tumor growth showed that TFAM loss resulted in greater tumor mass compared with control tumors. (D) Flow cytometry based cell cycle analysis showed that TFAM loss resulted in faster cycling while lower cell population in G0/G1 phase and more cells are distributed in S and G2M phases. (E) Annexin V based flow cytometric analysis was performed to determine the apoptotic status in TFAM silencing HNC cells. TFAM loss resulted in lower apoptotic percent in HNC cells. (F) TFAM loss led to increased migration in HNC cells by using transwell based cell motility assay. (G) Upregulation of half maximal inhibitory concentration (IC50) for chemotherapeutic agent CDDP, 5FU and Taxol was detected in response to TFAM loss in HNC cells. Data are presented as Mean \pm SEM $(N=3) .{ }^{*} p<0.05,{ }^{*} p<0.01,{ }^{* \star *} p<0.001$. 

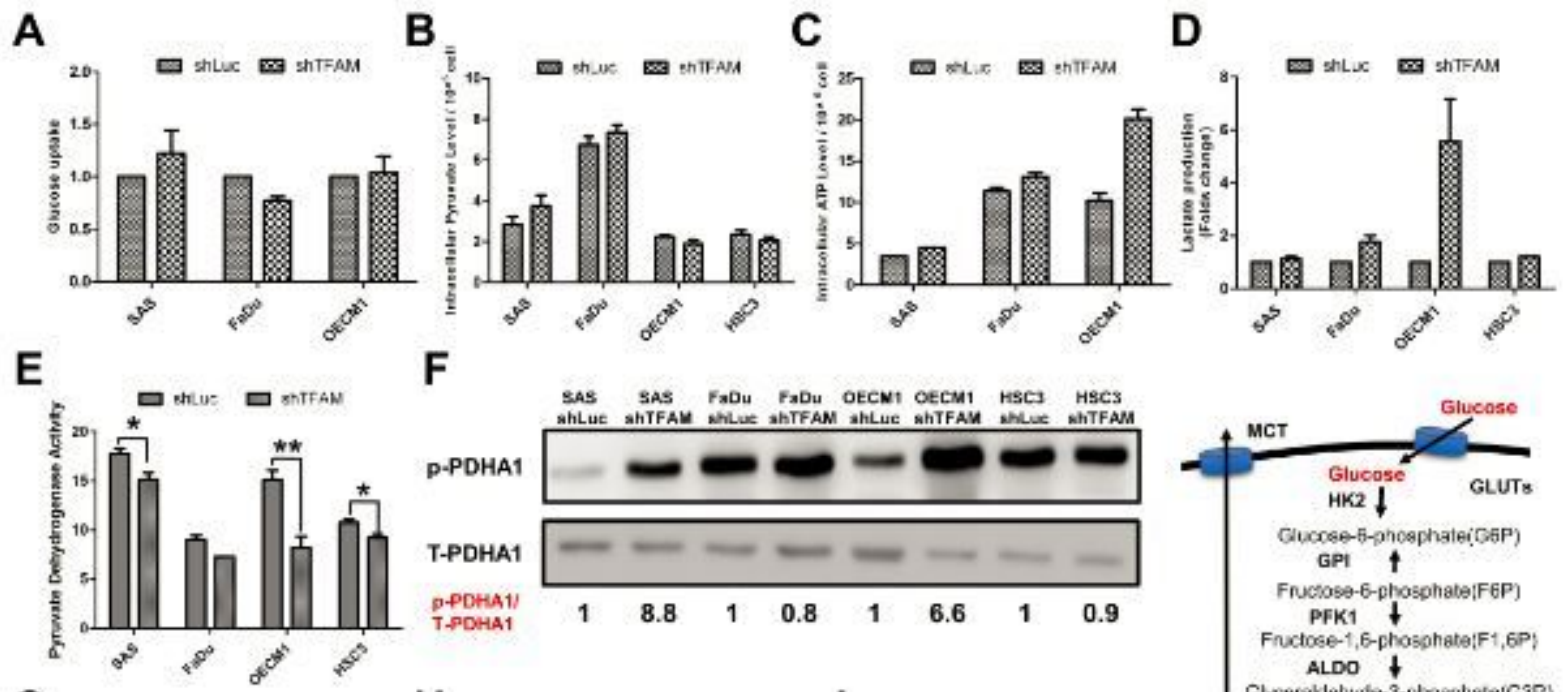

$\mathbf{F}$

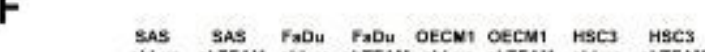
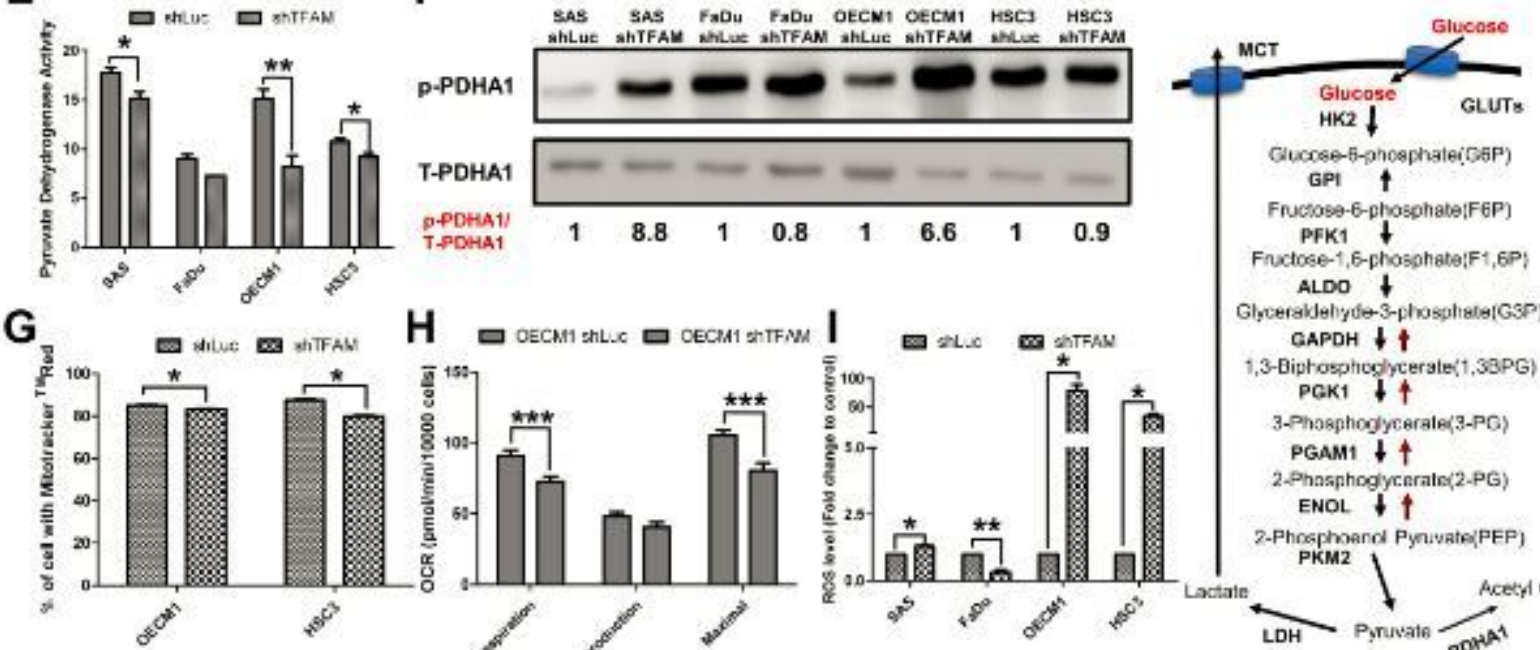

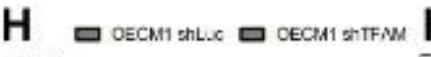

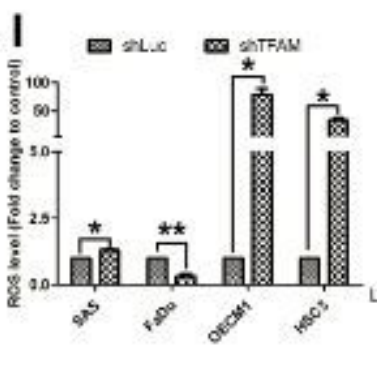

Glyceraldehyue-3-phosphisia|GSP) GAPDH $\downarrow \uparrow$

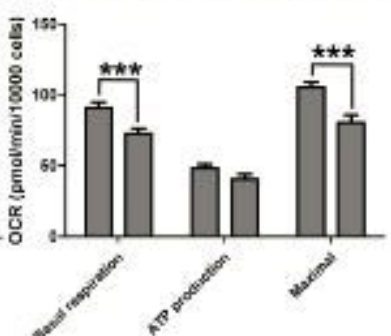

3-Biphosphoglycerate(1,38PG) PGK1 $\downarrow+$

3-Phosphogycerale〈3-PC)

PGAM1 $\downarrow \uparrow$

2.Phasphoglycerale(2-PG)

ENOL $\downarrow \uparrow$

2-Phosphoand Pynivates(PEF)

PKM2
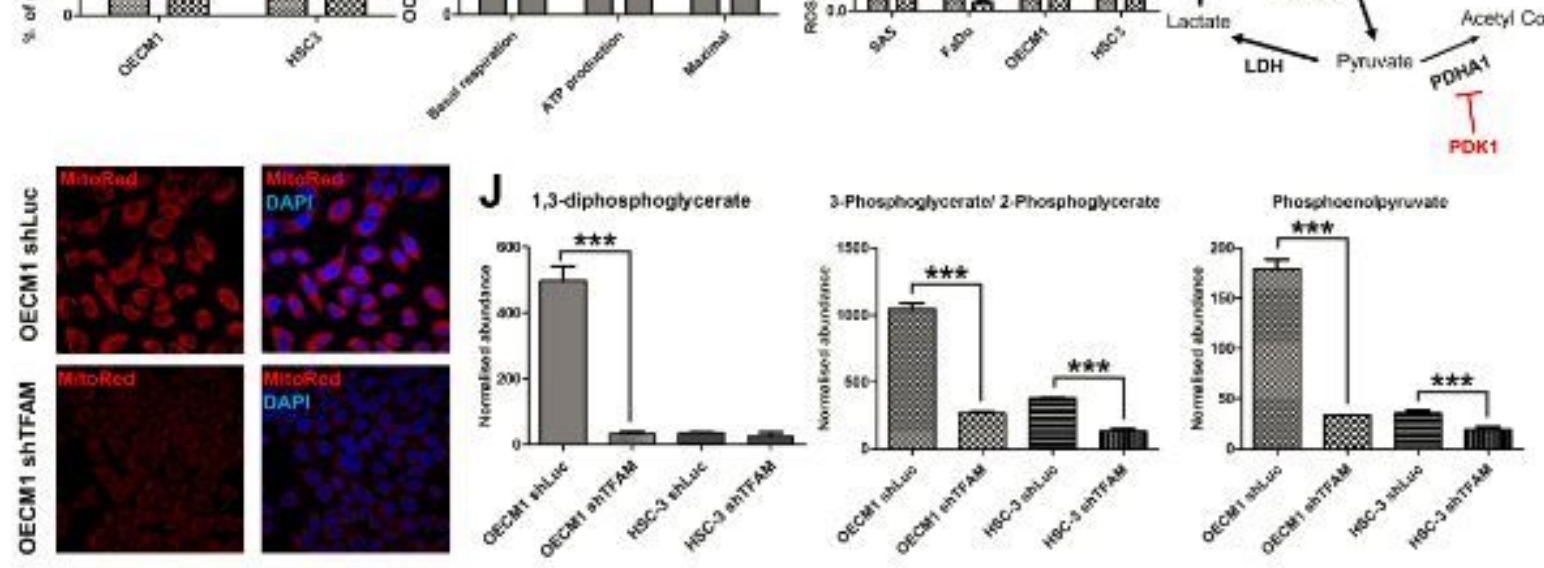

Figure 3

TFAM Silencing Elicits Metabolic Reprogramming in HNC Cells No significant changes of (A) Glucose uptake, (B) Intracellular pyruvate and (C) ATP levels were found in TFAM silencing HNC cells compared with control cells. (D) Increasing extracellular lactate levels and (E) significant decreased pyruvate dehydrogenase activity were detected in TFAM silencing HNC cells. (F) Western blot analysis further showed an increase of phosphorylated PDHA1 (inactive form) protein expression in TFAM silencing HNCs cells. (G) Flow cytometry and immunofluorescence staining analysis showed that TFAM loss led to decreased mitochondrial activity by Mitotracker Red staining in HNC cells. $(\mathrm{H})$ Seahorse bioanalyzer to determine basal, maximal respiration and ATP production showed a drop of respiration in TFAM silencing HNC cells; while (I) significant upregulation of intracellular ROS content using a flow cytometry based DCFDA assay for was detected in TFAM-silencing HNC cells. (J) Using LC-MS based metabolomic analysis for detection of glycolytic metabolites, it was shown that TFAM loss could result in decreased 
intracellular 1,3-diphosphoglycerate, 3-phosphoglycerate/2-phosphoglycerate and phosphoenolpyruvate abundance in HNC cells. Data are presented as Mean \pm SEM $(N=3) .{ }^{*} p<0.05,{ }^{* *} p<0.01,{ }^{* \star *} p<0.001$.

\section{Figure 4}

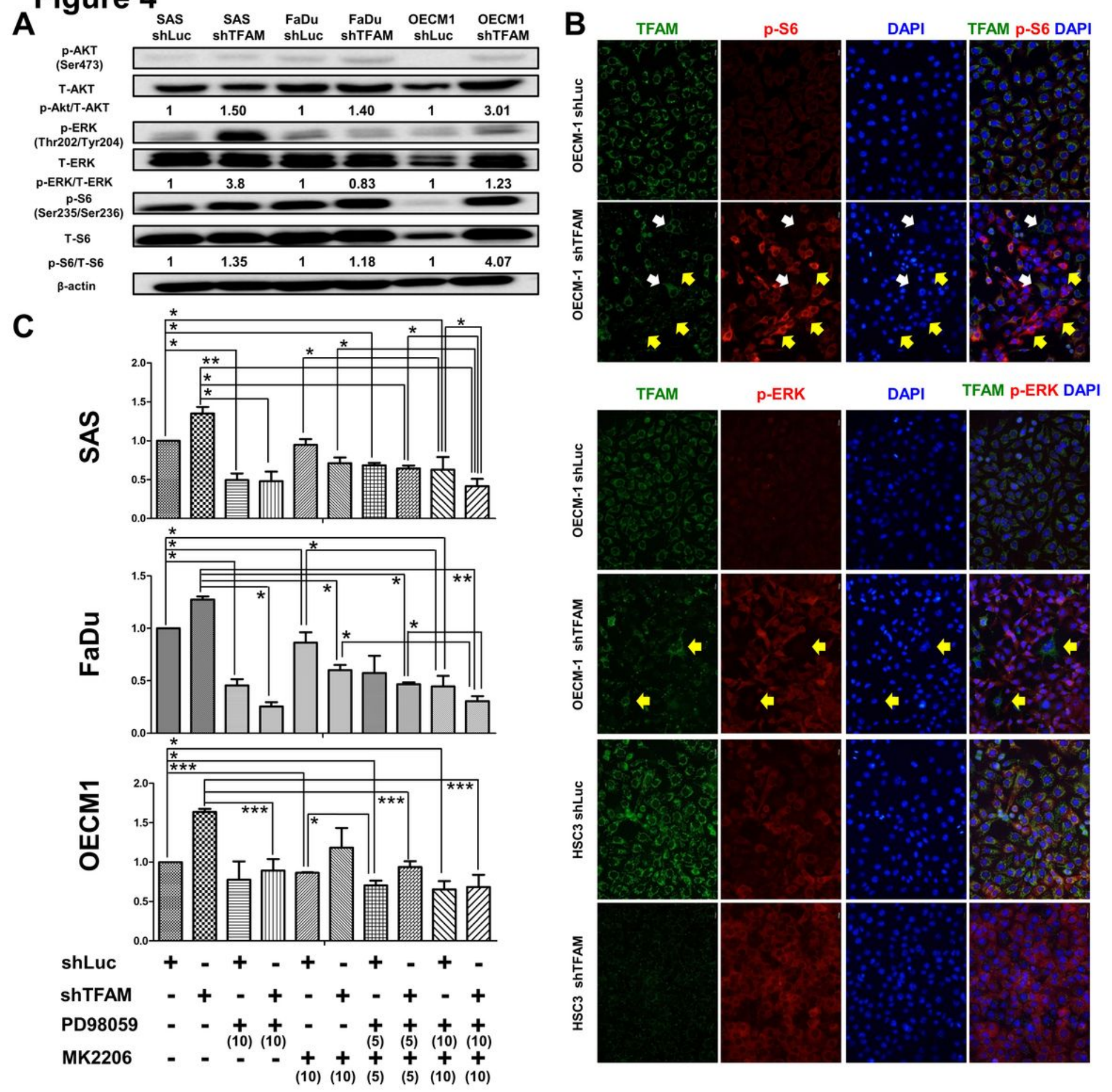

Figure 4

PKB/Akt and ERK Signaling Pathways Underlie TFAM Mediated Malignant Changes in HNC Cells (A) Western blot analysis showed increased expression of phosphorylated Akt (Ser473), phosphorylated p44/42 MAPK ERK1/2 (p-ERK, Thr202/Tyr204) and mTORC pathway effector phosphorylated S6 Ribosomal Protein (p-S6, Ser235/Ser236) in TFAM-silencing HNC cells. (B) Immunofluorescence staining 
analysis indicated that HNC cells with lower TFAM expression (yellow arrows) exhibited greater phosphorylated S6 and ERK expression as HNC cells with higher TFAM levels displayed lessp-S6 (while arrows). (C) Trypan blue exclusion assay for TFAM-silencing SAS, FaDu, OECM1 cells solely- or co-treated with PKB/Akt inhibitor MK2206 and ERK1/2 inhibitor PD98059 showed that PKB/Akt and ERK activities contribute to TFAM-mediated increased cell growth in a dose-dependent manner. Doses $(\mu \mathrm{M})$ of inhibitors are shown in parentheses. Data are presented as Mean \pm SEM $(N=3) .{ }^{*} p<0.05,{ }^{*} p<0.01,{ }^{*} *{ }^{*} p<0.001$.

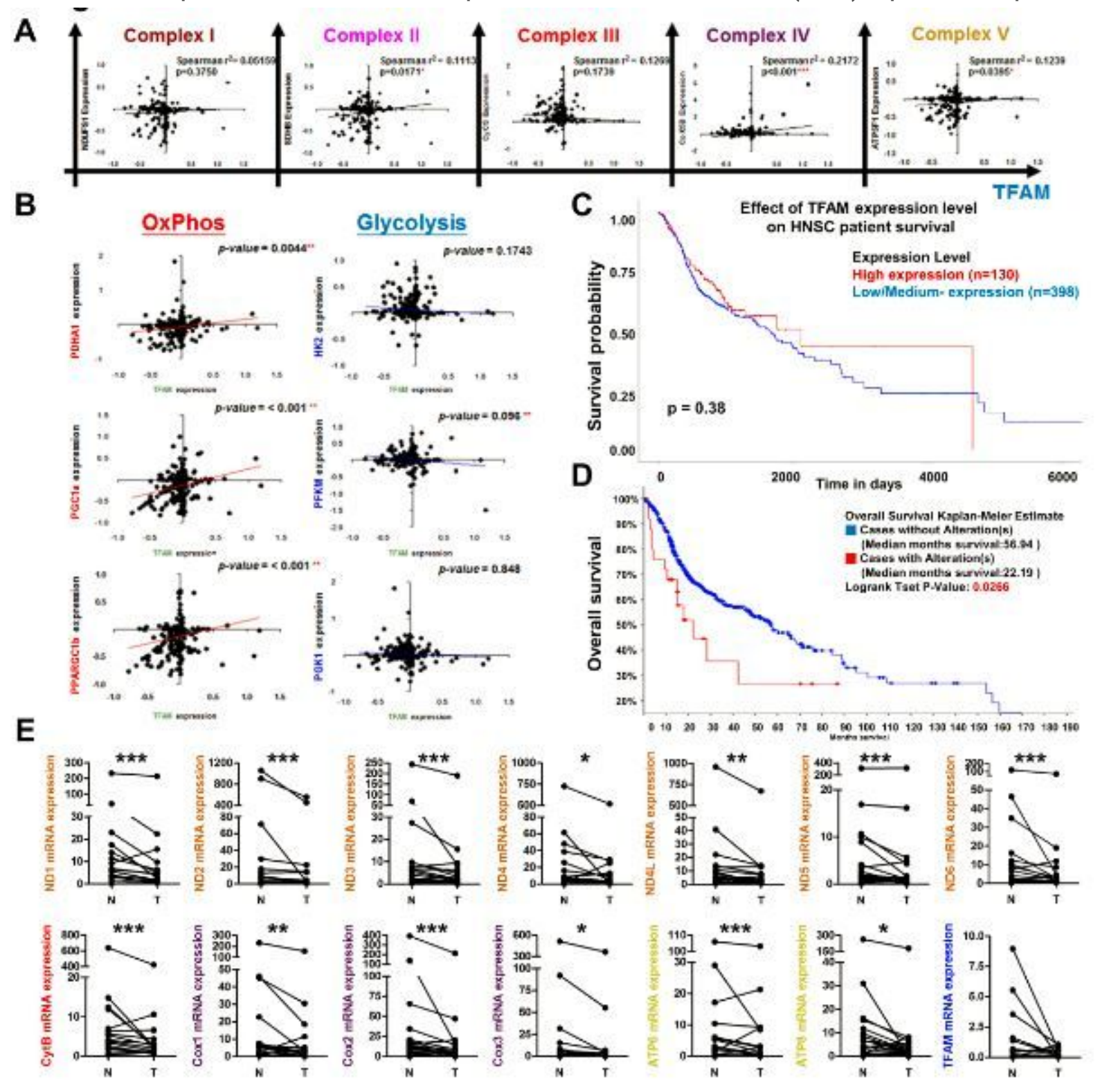

Figure 5

Clinical Impacts of TFAM and mtDNA-encoded ETC in HNCs (A) Positive correlations between TFAM and ETC subunits including Complex I: NDUFS1, Complex II: SDHB, Complex III: Cycs, Complex IV: Cox 5B, Complex V: ATP5F1 and (B) mitochondrial factor (PDHA1, PGC1a, PPARGC1 $\beta$ ) were detected in TCGA based database; whereas negative correlations between TFAM and glycolytic enzymes (HK2, PFKM, PGK1) were found in HNCs using The Cancer Genome Atlas (TCGA) database (N=273). Kaplan-Meier analysis for overall survival rates in HNC patients classified by (C) TFAM expression ( $N=519)$ and (D) 
TFAM genetic alteration(s) using TCGA database ( $\mathrm{N}=519)$. HNC cases with TFAM alteration(s) have significant poorer overall survival ( $\mathrm{N}=26$, median months survival: 22.19$)$ than $\mathrm{HNC}$ cases without TFAM alteration(s) ( $N=462$, median months survival: 56.94). (E) Real-time RT-PCR analysis detected mtDNA encoded ETC genes in HNC tumors ( $T$ ) and their corresponding adjacent normal tissues (N) from IRBapproved clinical specimens $(\mathrm{N}=15)$. Results showed significant decreased expression in $13 \mathrm{mtDNA}$ encoded ETC transcripts and decreasing TFAM mRNA expression in clinical tumorous tissues compared with their normal counterparts. Data are presented as Mean \pm SEM $(N=3) .{ }^{*} p<0.05,{ }^{*} p<0.01,{ }^{* \star *} p<0.001$.

\section{Supplementary Files}

This is a list of supplementary files associated with this preprint. Click to download.

- SupplementaryFigure.pdf

- SupplementaryFigure.pdf

- SupplementaryFigure.pdf

- SupplementaryTable.pdf

- SupplementaryTable.pdf

- SupplementaryTable.pdf 